\title{
VIRTUAL ROBUSTNESS EVALUATION OF TURBINE STRUCTURE ASSEMBLIES USING 3D SCANNER DATA
}

\author{
Anders Forslund \\ Chalmers University of Technology \\ Product and Production Development \\ SE-412 96 Göteborg, Sweden \\ anders.forslund@chalmers.se \\ Johan Lööf \\ Volvo Aero Corporation \\ 46181 Trollhättan, Sweden \\ johan.loof@volvo.com
}

\author{
Rikard Söderberg \\ Chalmers University of Technology \\ Product and Production Development \\ SE-412 96 Göteborg, Sweden \\ rikard.soderberg@chalmers.se \\ Alejandro Vega Galvez \\ Volvo Aero Corporation \\ 46181 Trollhättan, Sweden \\ alejandro.vega.galvez@volvo.com
}

\section{ABSTRACT}

In this paper, the functional robustness of a jet engine component is investigated. Located at the rear part of the engine, the Turbine Rear Structure (TRS) provides a support structure for the low-pressure shaft, while redirecting the exhaust flow from the low-pressure turbine to the exit nozzle. For larger engines, TRSs are fabricated assemblies consisting of cast, wrought and sheet metal parts.

In a case study, virtual tools are used to examine how geometrical variation in cast parts of the TRS assembly affects performance. Variation data are obtained by scanning cast parts in a 3D laser scanner. The resulting data are fed into a CAD model as surface point parameters. The parts are then assembled virtually using CAT software. The assemblies are subsequently fed into a simulation platform where they are meshed, and CFD and FEM are used to evaluate the structural and aerodynamic effects of the variation.

To quantitatively analyze the effects of variation, five cast parts with different geometrical variations are virtually assembled into 25 geometries and analyzed with respect to sixteen functional properties. Results show that geometric variation has a noticeable effect on performance.

We believe this approach to be a useful tool in engine design. Being able to virtually examine the geometrical robustness of a design in early phases reduces the need for redesign loops. This leads not only to faster and less expensive product development, but also to better and more reliable engine designs.
Keywords: Robust Design, 3D Scanner, Finite Element Analysis, Fluid Dynamics, Sensitivity Analysis.

\section{NOMENCLATURE}

CAD Computer Aided Design

CAT Computer Aided Tolerancing

CFD Computational Fluid Dynamics

FEM Finite Element Method

MDO Multidisciplinary Design Optimization

TRS Turbine Rear Structure

\section{PROBLEM DESCRIPTION}

For evaluation of jet engine components, simulation is preferred since it reduces the need for expensive physical testing. The testing is based on CAD models of the product, and as such, it is dependent on whether the CAD model is an accurate geometrical representation of the physical product. However, when a product is mass-produced, each realization of the product design will deviate from the nominal. Still, model validation is frequently performed based on comparison between simulations and output from single or repeated experiments. Extensive statistical experiments have often been deemed impractical because of the cost and time commitment associated with physical experiments [1]. 
A more efficient and less expensive way is to perform these quantitative statistical analyses in a virtual environment. Therefore, a case study was made to investigate virtual methods further. The purpose of this case study was to find a way to:

1. Validate $\mathrm{CAD}$ geometries against the manufactured products.

2. Quantify geometrical variation.

3. Examine the functional robustness of a given design, i.e. the effects of geometrical variation on performance.

A product whose function is insensitive to geometrical variation is defined as functionally robust [2]. In aero engine applications, functional robustness is often related to physical phenomena that are coupled. An example given in this paper is the thermal stress stemming from the heating of a turbine structure during flight. Since this problem is dependent on the geometry at hand, it is straightforward to realize that geometrical variation will affect structural strength, which will have an effect on product life length. However, geometrical variation will have another indirect effect as a change in the aero surface will affect the convective heat flow into the material, resulting in a different thermal expansion and life length.

Approaching the above problem requires the use of many engineering disciplines. For a deterministic evaluation of a nominal product, the common approach is for these analyses to be performed in different simulation environments by specialists in each field, with data being manually transferred between them. For robustness and sensitivity analyses, however, this process becomes ineffective and time-consuming [3]. In this paper, an automated, sequential process is suggested for capturing the problem, which allows for parameterizations to be propagated from one end of the analysis chain to the other. A method of combining different analysis methods into a multidisciplinary simulation platform is suggested. This method is then used to investigate the robustness of a generic loadcarrying rear frame, which is analyzed with respect to thermal stress as well as structural strength, aerodynamics, weight and manufacturability.

From a technical point of view, the suggested approach means a significantly improved ability to numerically simulate and optimize robustness of component designs with functionality criteria from principally different disciplines. From an industrial application point of view, the suggested approach provides a tool for including part variation in the early design face, rather than being treated downstream in the development process.

\subsection{Technical Background}

The commercial turbofan engines of today are designed to be fuel-efficient. This is accomplished by increasing the bypass ratio, which in turn implies large fan diameters. Modern engines are significantly larger than engines designed 30 years ago. The components inside the engine have also increased in size.

The turbine structures in the rear end of a jet engine have a range of functional criteria from various fields of engineering. They need to be able to withstand significant thermal and structural loads. In addition, to optimize fuel efficiency, they need to be as light and aerodynamic as possible. These functionality criteria must be balanced in order to obtain an optimal design.

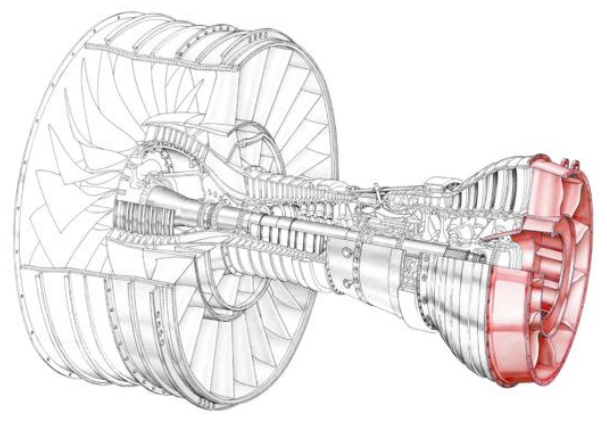

FIGURE 1: TURBINE REAR STRUCTURE

However, manufacturability criteria are often difficult to quantitatively assess in the design optimization process. As a result, it is often the case that designs optimized from a functionality perspective are expensive or unfeasible to realize in practice. To avoid this scenario, the functionality and manufacturability need to be balanced in order to find the truly optimal design [3]. One of the key limitations of manufacturability is geometrical variation, i.e. that the dimensions of a manufactured product deviate from the nominal geometry. Geometrical variation occurs at many stages [4]. Deviations in ingoing parts, as well as dislocations when placing parts in fixtures, propagate through the assembly, and ultimately affect the performance of the engine.

For small engines, TRSs are usually cast in one piece. For the larger engines of today, however, TRSs are welded assemblies consisting of cast, wrought and sheet metal parts. The ingoing parts all have some degree of geometrical variation. This part variation propagates through the fixturing and welding process into the final assembly. The assembly variation is dependent on part design, placement of fixturing points and welding sequence. By controlling these factors in an appropriate way, assembly variation can be suppressed.

\subsection{Theoretical Background - Robust Design}

Robust design is a methodology for designing products that are insensitive to variation. According to Phadke [5], product variation may stem from raw material variation, manufacturing variation and variation in product usage. Robust design aims at suppressing the effects of this variation without eliminating the variation itself. Robust design methodology was pioneered by Japanese statistician Genichi Taguchi [6, 7].

Robust Tolerance Design 
Robust tolerance design deals with geometrical variation in parts, fixtures and assemblies. A geometrically robust design is defined by Söderberg [4] as a design that fulfills its functional requirements and meets its constraints even when the geometry is afflicted with small manufacturing or operational variation.

Smith [2] defines robustness as functional insensitivity to stochastic variation. Therefore, how much variation in the assembled geometry that can be accepted depends on the functional requirements of the product. Lorin [8] divides geometrical robustness into three categories: part robustness, assembly robustness and functional robustness. The factors that define these characteristics and how they are related are visualized in Figure 2.

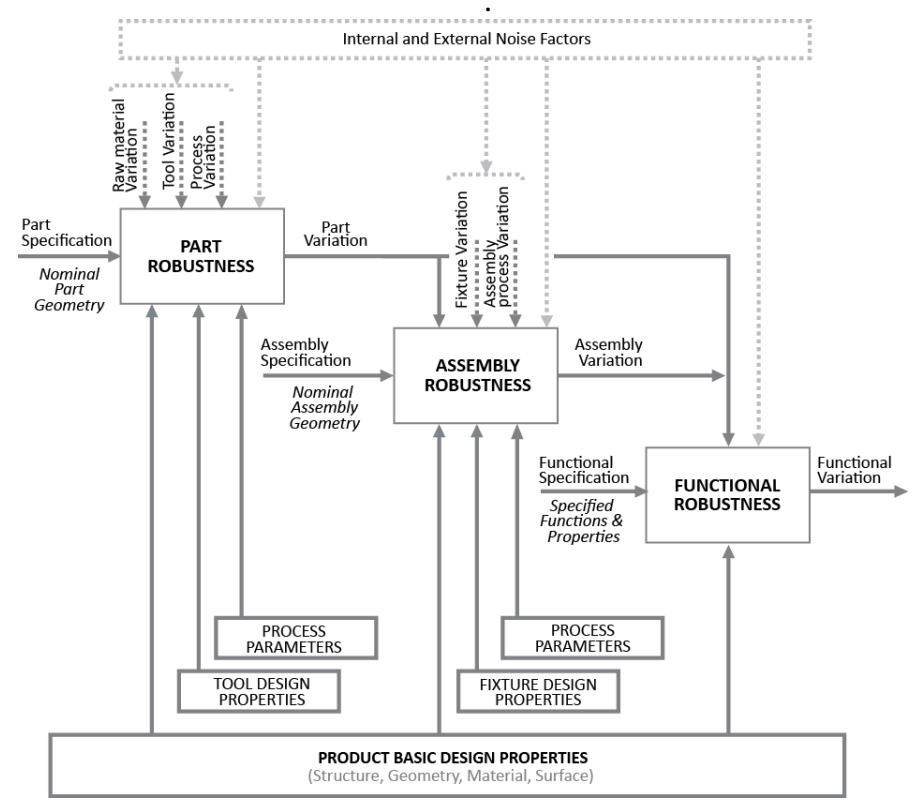

FIGURE 2: PRODUCT ROBUSTNESS [8]

A previous case study done on the same problem [9] investigated the effects of assembly variation on functional performance. This was done by applying variation in fixturing points, as proposed by Söderberg [4]. However, this approach only gives a approximate answer, as the exact nature of the variation in cast goods was not quantitatively examined.

\section{Locating Schemes}

The purpose of a locating scheme is to lock a part or a subassembly to its six degrees of freedom in space. Figure 3 shows an orthogonal 3-2-1 locating scheme. The points in the upper right body, the so-called A-points, control three degrees of freedom: translation in $\mathrm{Z}$, and rotation around $\mathrm{X}$ and $\mathrm{Y}$. The two points in the lower left figure, the B-points, control two degrees of freedom: translation in $\mathrm{Y}$ and rotation around $\mathrm{Z}$. Finally, the C-point in the lower right figure controls the translation in X. [10]

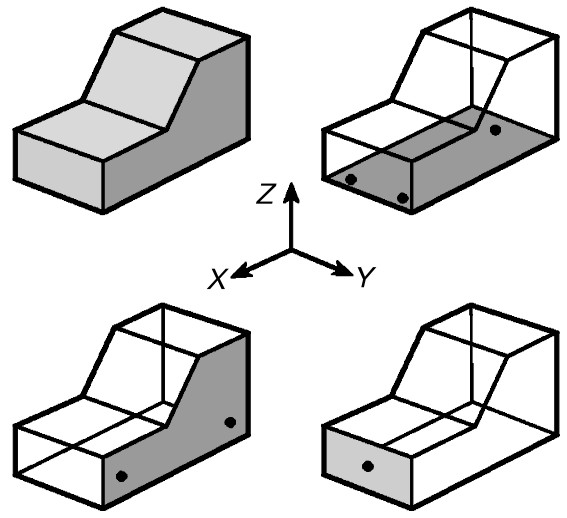

FIGURE 3: 3-2-1 LOCATING SCHEME

When attaching a part to an assembly, all six degrees of freedom need to be locked. The part's local positioning scheme, or local p-frame, should be matched by a target p-frame, as shown in Figure 4.

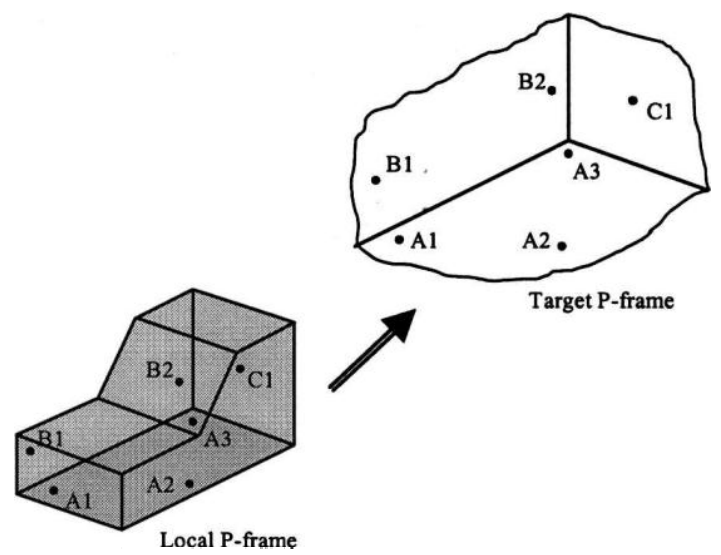

FIGURE 4: POSITIONING OF A PART

Applying variation to the locating points will then affect the positioning of the parts, and therefore, the selection of locating points should be made to minimize the effects of variation on the part position stability [4]. Automated ways of optimizing locating schemes have been put forth [11], as well as methods for optimal allocation tolerances on these locating points [12].

\subsection{Other Related Work}

Robust design is seen by some [13] as a subset of response surface methodology, which in turn is one of the methods employed in the field of Multidisciplinary Design Optimization (MDO), an area of much research for aerospace applications [14]. According to Havakechian [15], a future trend in MDO is to cover not only aerodynamic performance of turbines and compressors, but also geometrical requirements, mechanical integrity and manufacturing costs. Dornberger [16] suggests adding disciplines such as life cycle costs, product life cycle time, weight, emissions and heat transfer to the equation.

Robust design practices have been applied to FEM [17] and CFD [18] , as well as for variation propagation control in aero- 
engine assembly [19]. In this paper, the connection between these fields of science is investigated in one coupled, multidisciplinary problem.

\section{CASE STUDY}

This section presents a case study that connects geometrical variation in a turbine structure with its functionality, thus investigating the functional robustness of a given turbine structure design.

The turbine structure is shown in Figure 5. The structure is a fabricated assembly, consisting of a number of guide vane mount sections and corresponding hub sections. Two of the mount sections have mount lugs, which are used to attach the aft section of the engine to the aircraft pylon. Ring-shaped flanges are attached to the front and back of the shroud. The parts are placed in fixtures and welded together.

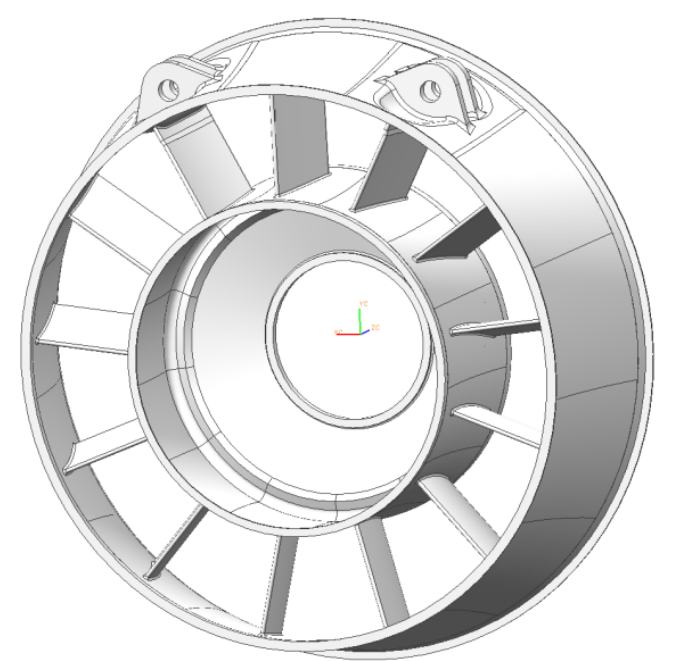

FIGURE 5: TURBINE STRUCTURE CAD MODEL

In this case study the design space was limited to the assembly of the two mount lug sections. The sections are mounted in fixtures and welded to the assembly.

The locating scheme of the T-section is shown in Figure 6.

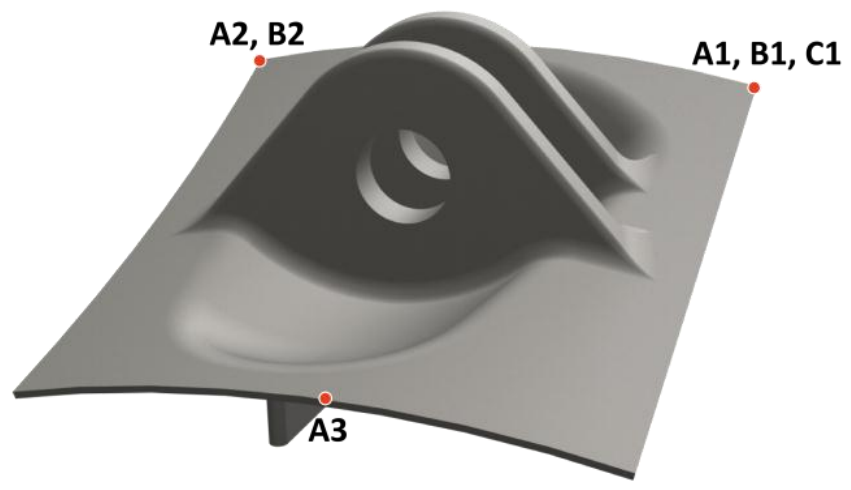

FIGURE 6: PART LOCATING POINTS

\subsection{Geometry generation}

Laser 3D scanning makes it possible to analyze complex geometries on a large scale. Today, 3D scanners typically have measurement ranges around \pm 5 to $\pm 250 \mathrm{~mm}$, and accuracies at about 1 part in 10,000 and measurement frequency of $40 \mathrm{kHz}$ or higher [20].

1. Although automated approaches exist that automatically yield CAD parts from scanner data, there are some limitations:

2. 3D scanning only captures non-occluded surfaces, thus only yielding information on a subset of the geometry.

3. The scanned geometry lacks information of abstract concepts of geometrical shape. A CAD model differentiates between spheres, cylinders, rectangles, splines, etc. A laser scanner returns objects as generic shapes defined by a set of data points. A laser-scanned model is not as easily parameterized as a CAD model.

This problem was resolved by mapping the point cloud data to a set of design point parameters. These point parameters corresponded to certain points used in the original $\mathrm{CAD}$ generation. In this way, a parameterized CAD model, similar to the original model, could be obtained using the same design practices as in the original model.

The geometry was interpolated from the design point parameters. Spline interpolation [21] was used to create curves from the points. Splines are piecewise-smooth polynomial functions that are commonly used in CAD applications for curve fitting. The splines were second-degree. To interpolate between $n$ points, $(n-2)$ segments were used. This yields an exact solution where the curve touches all points.

The areas between these curves were then swept to generate surface models, which was subsequently uniformly thickened. Separate surfaces were created for the guide vane, the shroud and the mount lugs. The guide vane surface was extended using a support curve so that it fully intersected with the shroud.

As the measurement points were largely focused on the aero side of the surface, the opposing surface was merely the result of the uniform thickening operation. As a final step, the thickened surfaces were trimmed against each other and united into one solid body. All the steps of the process are illustrated in Figure 7. The original design CAD model was used as a reference, as the scanner data can be interpreted in different ways. 


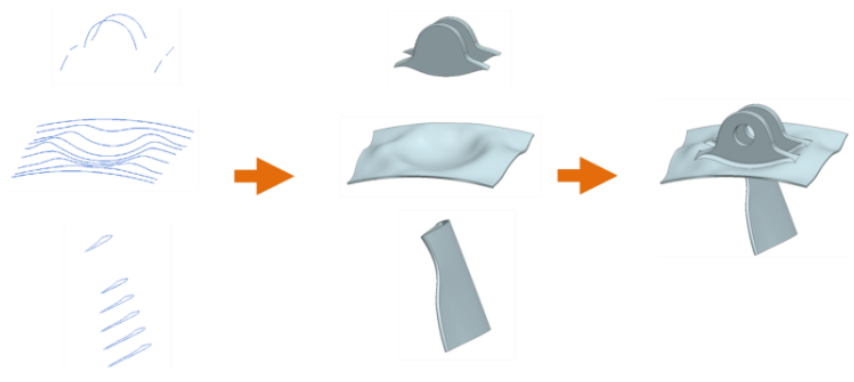

FIGURE 7: GENERATING THE MODEL FROM MEASUREMENT DATA

The model was then virtually assembled, using the 3-2-1 locating scheme defined in Figure 6. Upon this, a virtual welding procedure was performed to connect the mount lugs to the assembly. This welding procedure consisted of sweeping surfaces to create a solid weld between the interfacing parts. A tangential condition is set on connecting surfaces to get a smooth transition, as we have previously observed [9] that nonsmooth transitions concentrate stresses in corners. Although this procedure is hardly a realistic depiction of the welding process, the final result is nevertheless a fully connected assembly that can be used for applying variation to parts. Figure 8 shows a realization of such an assembly, when variation is applied on the locating points. The variation, which can be seen on the connecting edges, is exaggerated for visualization purposes.

Two different CAD geometries were created, one for CFD and one for FEM. The CFD geometry made use of the periodic nature of the aero surface, and modeled only a sectional piece containing one guide vane. The FEM model contained the entire geometry.

\subsection{Integrated Simulation Platform}

The turbine structure has a range of functionality criteria from various fields of engineering. In this case study, an integrated simulation platform was used to examine these multidisciplinary criteria. Figure 9 shows the workflow of the platform.

The platform uses the umbrella software Ansys Workbench, where parameterized CAD models created in NX can be batchprocessed through meshing into CFD and FEM analyses. The process is fully automated and follows the traditional workflow for verification of turbine structures.

\section{Meshing}

Meshing was done using automated meshing algorithms. Separate meshes were used for the CFD and FEM analysis.

The CFD mesh model used a $30^{\circ}$ sectional model with periodic boundary conditions. This significantly reduces simulation time compared to a full $360^{\circ}$ degree model. The mesh contained about one million hexahedral cells, with a finer mesh close to the walls. The mesh density was set to ensure sufficient conversion. In the simulation, a realizable K-epsilon model with enhanced wall functions was used.

The FEM mesh was based on the $360^{\circ}$ degree model, with roughly one million tetrahedral cells. A nonlinear steady state solver was used.

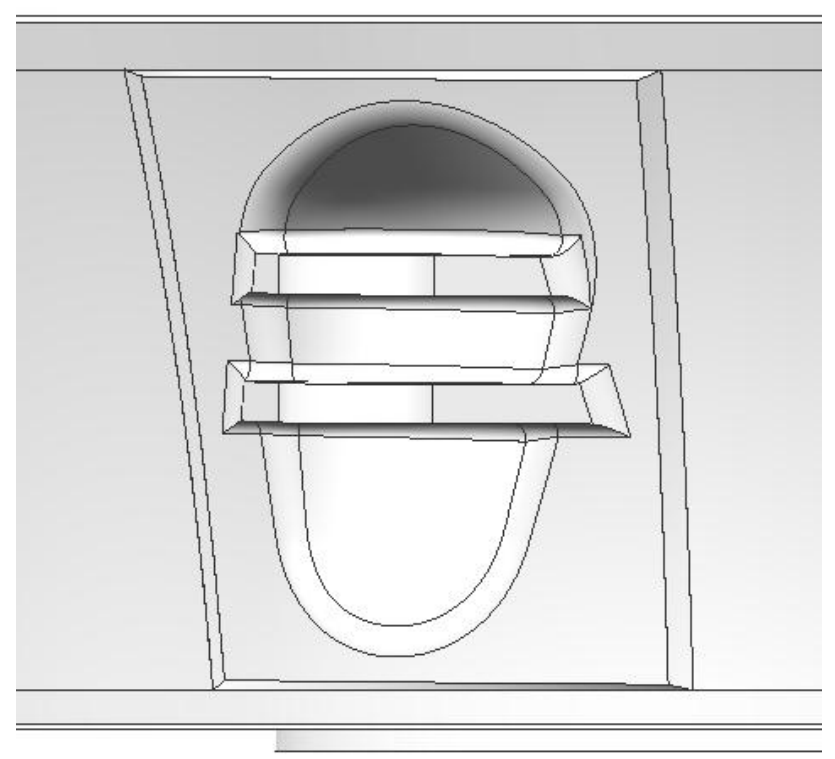

FIGURE 8: ASSEMBLY VARIATION CAN BE SEEN IN THE CONNECTING EDGES 


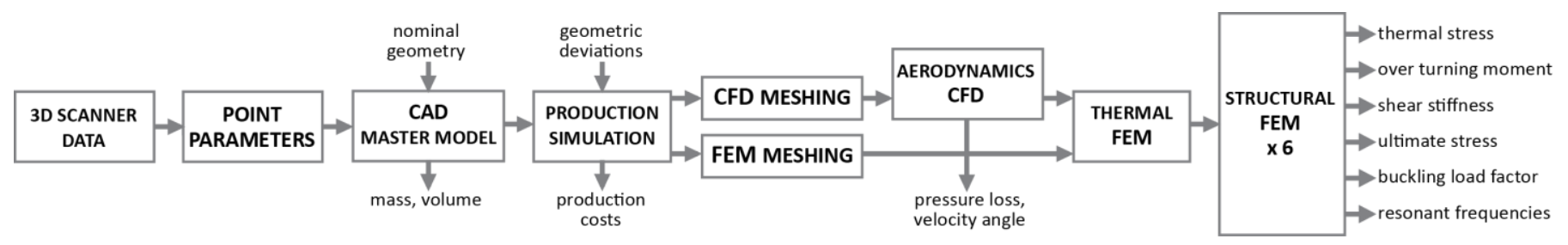

FIGURE 9: INTEGRATED SIMULATION WORKFLOW

\section{ANALYSES}

Eight different tests were carried out:

1. Weight analysis - calculates structural weight. The weight information was extracted directly from the CAD master model.

2. Aerodynamics analysis - evaluates the aerodynamic performance of the part. Specifically, the pressure loss over the trs and the velocity angle at the outlet are calculated. Further, aero surface temperatures are calculated and fed into the subsequent thermal analysis.

3. Thermal analysis - calculates the material temperature from given boundary surface temperatures. The results of the thermal analysis are used to calculate thermal stress.

4. Thermal stress - The recurring thermal loads on the frame create large stresses in the material. This is a limiting factor for product life. Consequently, the thermal stress gives an indication of estimated life. Centerline shift, the movement of the motor shaft centerline because of thermal expansion, was also calculated.

5. Ultimate stress - assesses whether the turbine structure can withstand extreme events, such as a loss of a fan or turbine blade, or a wheels-up landing. The engine does not need to be operational after such an event, but the engine must not separate from the wing, and no parts should be lost. Ultimate stress is measured on the primary and secondary load paths.
6. Shear compliance - calculating the inverse of the stiffness of the product, when a unit load is acting on the bearing housing. Compliance is chosen instead of stiffness in order to consistently define the output as something that should be minimized.

7. Overturning moment - similar to shear compliance, but instead of a force, a torque on the bearing housing around the pitch axis.

8. Modal analysis - calculates the Eigen modes of the structure. The frequencies of these Eigen modes should be far from the engine RPM to prevent resonance of mechanical loads, something that can have severe consequences.

\section{Simulation times}

The average simulation time for the entire workflow was approximately 90 minutes. The CFD analysis involved approximately 100 iterations. In the simulation, a realizable $\mathrm{K}$ epsilon model with enhanced wall functions was used. As each iteration took a bit less than one minute, the total simulation time for the CFD analysis was around 60-65 minutes.

The six FEM analyses were less computationally intensive than the CFD calculation. The modal analysis took the most time - approximately 20 minutes. The other five analyses took less than 10 minutes.

As 25 different geometries were analyzed, the total simulation time was 45 hours - a little bit less than two days. 
TABLE 1: MONTE CARLO SIMULATION RESULTS

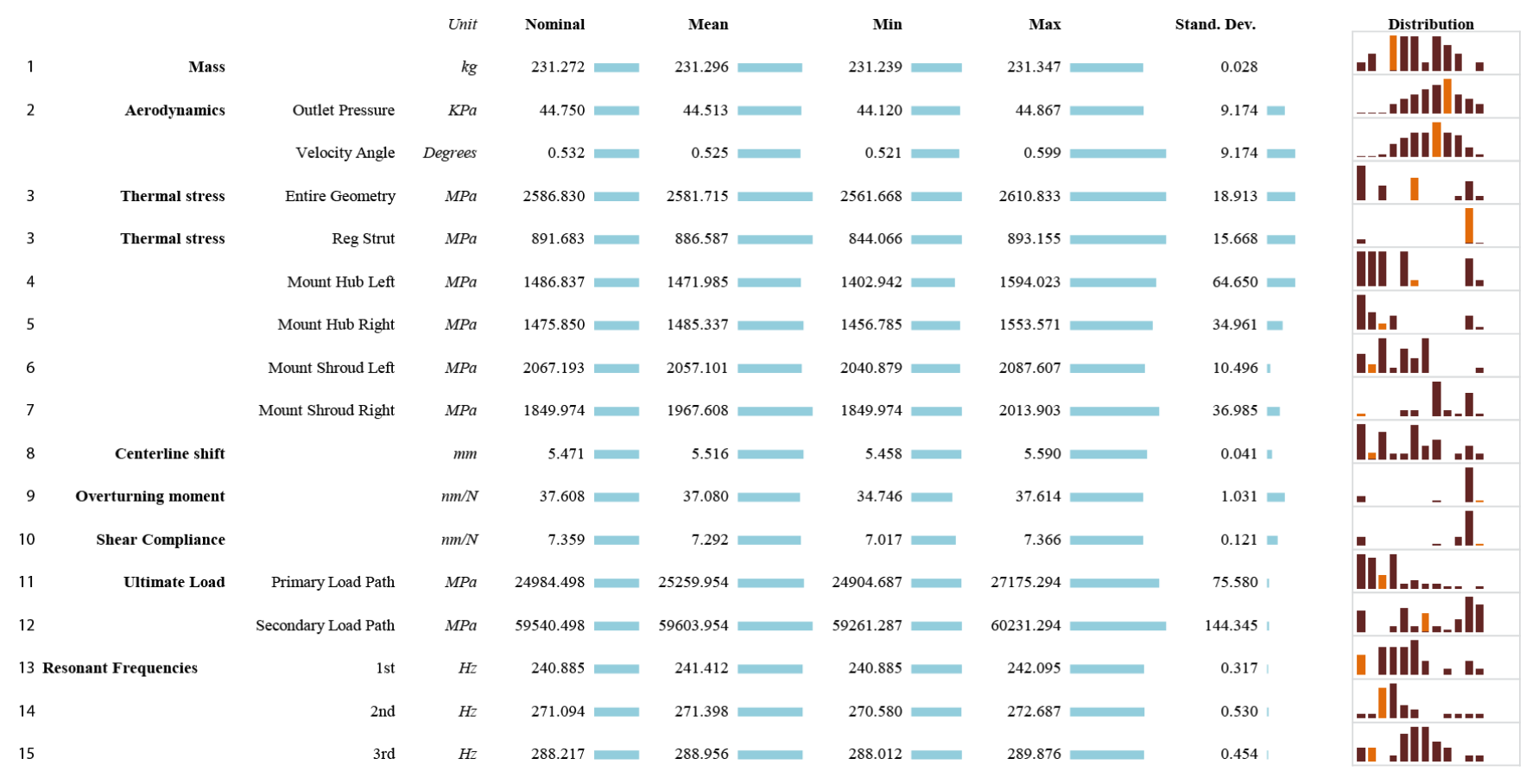

\section{RESULTS}

Table 1 shows the results from the simulations. Some of the outputs are almost unaffected by the geometrical variation of the cast goods. Mass, centerline shift and resonant frequencies all show variation of less than $1 \%$.

From the histograms on the right side of the figure, we note that the distribution functions are very different from each other. For a perfectly linear input/output relation, Gaussian input parameters should yield a Gaussian output. The mass and centerline shift seems to be roughly linear. Even the aerodynamics results, which are calculated using nonlinear equations, appear to follow a Gaussian bell curve.

For a fully optimized geometry, each variation should yield a result that is worse than the nominal. As all outputs (except the resonant frequencies) are desirable to minimize, an optimized design should have the orange nominal bars to the left side of the histogram. This is true for many of the outputs, like the mount shroud stresses and centerline shift.

The outlet pressure is varying in the range of a quarter of a percent. This is significant variation as it directly affects engine thrust and fuel economy. This underlines the importance of controlling geometric variation.

Looking at the histograms for the thermal stresses, it's clear that they are clustered around certain ranges. Although 25 different simulations were performed, only five different geometries were used. Table 10 looks more closely at the thermal stresses in each of the hub and shroud components. The four sub-tables are color-coded independently. Inspecting this table, it is clear that left hub stresses are mainly dependent on the variation in the left hub, and vice versa. This seems logical. The shrouds show the same trend, although not as clearly, and with more outliers. Thus, combinatorial effects are not negligible, and the stress levels need to be investigated simultaneously.

TABLE 2: THERMAL STRESSES IN HUB AND SHROUD SECTIONS

\begin{tabular}{|c|c|c|c|c|c|c|c|c|c|c|c|}
\hline & & L1 & $\mathrm{L} 2$ & L3 & L4 & L5 & L1 & $\mathrm{L} 2$ & L3 & $\mathrm{L} 4$ & L5 \\
\hline \multirow{5}{*}{$\stackrel{\ominus}{3}$} & $\mathrm{R} 1$ & 1431.3 & 1411.3 & 1494.5 & 1588.8 & 1437.8 & 1472.2 & 1470.6 & 1471.8 & 1471.1 & 1470.3 \\
\hline & $\mathrm{R} 2$ & 1432.5 & 1406.9 & 1486.4 & 1590.8 & 1440.0 & 1458.4 & 1457.0 & 1461.5 & 1457.4 & 1456.8 \\
\hline & R3 & 1430.8 & 1402.9 & 1473.3 & 1594.0 & 1454.2 & 1460.0 & 1458.5 & 1459.0 & 1458.8 & 1458.7 \\
\hline & $\mathrm{R} 4$ & 1433.1 & 1406.9 & 1486.2 & 1587.9 & 1441.0 & 1552.8 & 1550.1 & 1549.9 & 1553.3 & 1553.6 \\
\hline & R5 & 1432.8 & 1406.7 & 1486.4 & 1587.9 & 1440.4 & 1490.7 & 1481.4 & 1862.1 & 1490.4 & 1489.1 \\
\hline \multirow{6}{*}{ 总 } & $\mathrm{R} 1$ & 2058.3 & 2066.7 & 2057.0 & 2043.3 & 2048.5 & 1929.3 & 1923.2 & 1964.5 & 1925.2 & 1921.0 \\
\hline & $\mathrm{R} 2$ & 2051.1 & 2060.4 & 2052.5 & 2040.9 & 2066.3 & 2007.5 & 2000.7 & 2008.4 & 2003.5 & 1999.4 \\
\hline & R3 & 2053.1 & 2063.1 & 2051.1 & 2087.6 & 2050.3 & 1961.1 & 1956.6 & 1958.1 & 1958.0 & 1955.9 \\
\hline & R4 & 2059.5 & 2063.7 & 2052.4 & 2040.9 & 2067.1 & 2013.9 & 1966.5 & 1986.2 & 2009.4 & 2005.3 \\
\hline & R5 & 2059.9 & 2061.3 & 2053.6 & 2041.6 & 2067.1 & 1972.1 & 1978.9 & 1646.2 & 1967.8 & 1967.5 \\
\hline & & & \multicolumn{2}{|c|}{ Left } & & & & \multicolumn{2}{|c|}{ Right } & & \\
\hline
\end{tabular}



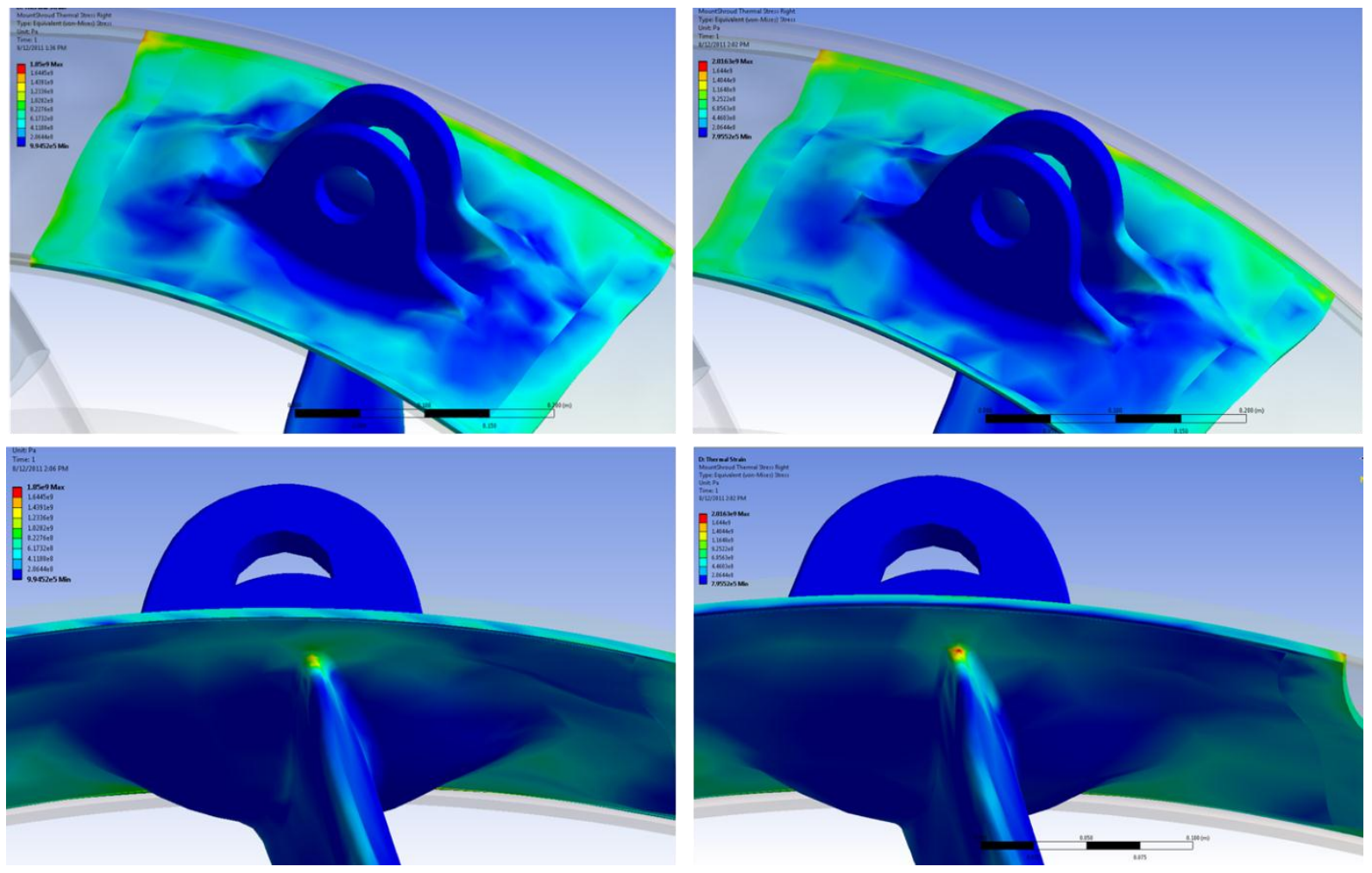

FIGURE 10: THERMAL STRESS, NOMINAL AND MAX, RIGHT MOUNT SHROUD

Figure 10 shows the nominal and maximum thermal strain for the right mount shroud. There is an $8 \%$ stress increase between the nominal (which is also the minimum) and the maximum case. For the nominal case, the max stress occurs in the far left corner, as shown in the top left figure. However, for the maximum case, the max stress occurs in the shroud-vaneblend.

\section{CONCLUSIONS AND DISCUSSION}

The case study underlines the fact that an integrated simulation platform is an extremely useful tool in engine design.

The results showed an evident variation in some of the functional characteristics as a result of geometrical variation. The conclusion should be drawn that geometrical variation and its effects cannot be neglected in product development working only with nominal geometry in simulation is insufficient for assessing real-world performance.

Interestingly, some of the deviating geometries showed better aerodynamic characteristics than the nominal geometry. This suggests that the nominal aero surface of the original design is suboptimal, and that some of the deviating geometries could serve as a base for a redesign. This should be investigated further, perhaps by implementing optimization techniques such as meta-modeling or genetic algorithms.

An overall trend is that stresses are concentrated in the connecting edges between objects. This has previously been identified as a problem area [9], but was thought to be an effect of a deficient CAD modeling approach - non-smooth edges were identified as causing this. The model presented in this paper only uses smooth edges in the model. Although this alleviates the effects significantly, stresses still concentrate around the edges. As these edges are welded together, a simulation that includes welding deformation would be desirable in order to account for changes in material properties and inner stresses, With recent advances in welding simulation techniques [3], it should be possible to link welding simulation to the platform.

As the thermal stress of the mount shrouds is a limiting factor of product life length, a large variation of stresses implies large deviations in life length between individual manufactured products. Such quality inconsistencies should best be avoided.

Another limitation of the simulation is that the virtual assembly was done with rigid components. In reality, the components exhibit some non-rigid behavior, the effects of which remain unassessed.

Further, another difficult thing to assess is the human factor in assembly. The assembly process is not fully automated, and the experience of the factory worker plays an important role in the final results. The experienced tweaking and turning of the assembly done by hand is perhaps the hardest thing to model on a computer. 


\section{ACKNOWLEDGEMENTS}

The work done in this paper was enabled with the expertise of the skillful people at Volvo Aero Corporation and Chalmers University of Technology. We would also like to acknowledge VINNOVA, the Swedish Agency for Innovation Systems, through the Swedish National Aeronautics Research Program - NFFP for their financial support.

\section{REFERENCES}

[1] Chen, W., Baghdasaryan, L., Buranathiti, T., and Cao, J., 2004, "Model Validation via Uncertainty Propagation and Data Transformations," AIAA Journal, 42(7), pp. 1406-1415.

[2] Smith, J., and Clarkson, P. J., 2005, "A method for assessing the robustness of mechanical designs," J Eng Design, 19(5), pp. pp. 493-509.

[3] Runnemalm, H., Tersing, H., and Isaksson, O., "Virtual Manufacturing of Light Weight Aero Engine Components," Proc. ISABE 2009.

[4] Söderberg, R., and Lindkvist, L., 1999, "Computer Aided Assembly Robustness Evaluation," J Eng Design, 10(2), pp. pp. 168-181.

[5] Phadke, M. S., 1989, Quality Engineering Using Robust Design, P T R Prentice-Hall Inc., Englewood Cliffs, New Jersey.

[6] Taguchi, G., Chowdhury, S., and Wu, Y., 2005, Taguchi's Quality Engineering Handbook, John Wiley \& Sons Inc., Hoboken, New Jersey.

[7] Taguchi, G., Elsayed, E. A., and Hsiang, T., 1989, Quality engineering in production systems, McGraw-Hill Book Company, Singapore.

[8] Lorin, S., Forslund, K., and Söderberg, R., 2010, "Investigating the Role of Simulation for Robust Plastics Design," Norddesign 2010, A. Dagman, ed.Göteborg, Sweden, pp. 185-194.

[9] Forslund, A., Söderberg, R., Isaksson, O., and Lööf, J., "Multidisciplinary Robustness Evaluation of Aero Engine Structures," Proc. ISABE 2011.

[10] Söderberg, R., Lindkvist, L., and Carlsson, J., 2006, "Virtual Geometry Assurance for Effective Product Realization," 1st Nordic Conference on Product Lifecycle Management - NORDPLM'06Göteborg.

[11] Lööf, J., Lindkvist, L., and Söderberg, R., "Optimizing Locator Positions to Maximize Robustness in Critical Product Dimensions," Proc. ASME Intenational Design Engineering Technical Conferences.

[12] Lööf, J., Hermansson, T., and Söderberg, R., 2007, "An efficient solution to the discrete least-cost tolerance allocation problem with general loss functions," Models for Computer Aided Tolerancing in Design and Manufacturing, J. K. Davidson, ed., Springer, Dordrecht, The Nederlands, pp. pp. 115-124.

[13] Myers, R. H., and Montgomery, D. C., 2002, Response surface methodology : process and product optimization using designed experiments, J. Wiley, New York.
[14] Sobieszczanski-Sobieski, J., and Haftka, R. T., 1997, "Multidisciplinary Aerospace Design Optimization: Survey of Recent Developments," Struct Optimization, 14(1), pp. 1-23.

[15] Havakechian, S., and Greim, R., 1999, "Aerodynamics design of 50 percent reaction steam turbines," P I Mech Eng CJ Mec, 213(1), pp. 1-25.

[16] Dornberger, R., Büche, D., and Stoll, P., "Multidisciplinary Optimization in Turbomachinery Design," Proc. ECCOMAS 2000.

[17] Chinchalkar, S., and Taylor, D. L., 1994, "Geometric Uncertainties in Finite-Element Analysis," Comput Syst Eng, 5(2), pp. 159-170.

[18] Putko, M. M., Taylor, A. C., Newman, P. A., and Green, L. L., 2002, "Approach for Input Uncertainty Propagation and Robust Design in CFD using Sensitivity Derivatives," J Fluid Eng-T Asme, 124(1), pp. 60-69.

[19] Yang, Z., Hussein, T., Popov, A. A., and McWilliam, S., "Novel Optimization Technique for Variation Propagation Control in an Aero-Engine Assembly," Proc. IMechE.

[20] Chen, F., Brown, G. M., and Song, M. M., 2000, "Overview of Three-Dimensional Shape Measurement Using Optical Methods," Opt Eng, 39(1), pp. 10-22.

[21] De Boor, C., 2001, A practical guide to splines : with 32 figures, Springer, New York. 